ORIGINAL ARTICLE

\title{
Childhood trauma predicts antidepressant response in adults with major depression: data from the randomized international study to predict optimized treatment for depression
}

\author{
LM Williams $^{1,2,5}$, C Debattista ${ }^{1,5}$, A-M Duchemin $^{3}$, AF Schatzberg $^{1}$ and CB Nemeroff ${ }^{4}$
}

Few reliable predictors indicate which depressed individuals respond to antidepressants. Several studies suggest that a history of early-life trauma predicts poorer response to antidepressant therapy but results are variable and limited in adults. The major goal of the present study was to evaluate the role of early-life trauma in predicting acute response outcomes to antidepressants in a large sample of well-characterized patients with major depressive disorder (MDD). The international Study to Predict Optimized Treatment for Depression (iSPOT-D) is a randomized clinical trial with enrollment from December 2008 to January 2012 at eight academic and nine private clinical settings in five countries. Patients $(n=1008)$ meeting DSM-IV criteria for MDD and 336 matched healthy controls comprised the study sample. Six participants withdrew due to serious adverse events. Randomization was to 8 weeks of treatment with escitalopram, sertraline or venlafaxine with dosage adjusted by the participant's treating clinician per routine clinical practice. Exposure to 18 types of traumatic events before the age of 18 was assessed using the Early-Life Stress Questionnaire. Impact of early-life stressors_overall trauma 'load' and specific type of abuse-on treatment outcomes measures: response: ( $\geqslant 50 \%$ improvement on the 17 -item Hamilton Rating Scale for Depression, $\mathrm{HRSD}_{17}$ or on the 16 -item Quick Inventory of Depressive Symptomatology_Self-Rated, QIDS_SR 16 ) and remission (score $\leqslant 7$ on the HRSD ${ }_{17}$ and $\leqslant 5$ on the QIDS_SR 16 ). Trauma prevalence in MDD was compared with controls. Depressed participants were significantly more likely to report early-life stress than controls; $62.5 \%$ of MDD participants reported more than two traumatic events compared with $28.4 \%$ of controls. The higher rate of early-life trauma was most apparent for experiences of interpersonal violation (emotional, sexual and physical abuses). Abuse and notably abuse occurring at $\leqslant 7$ years of age predicted poorer outcomes after 8 weeks of antidepressants, across the three treatment arms. In addition, the abuses occurring between ages 4 and 7 years differentially predicted the poorest outcome following the treatment with sertraline. Specific types of early-life trauma, particularly physical, emotional and sexual abuse, especially when occurring at $\leqslant 7$ years of age are important moderators of subsequent response to antidepressant therapy for MDD.

Translational Psychiatry (2016) 6, e799; doi:10.1038/tp.2016.61; published online 3 May 2016

\section{INTRODUCTION}

Globally, 405 million people experience depression and depression is a leading contributor to disability and lost productivity. ${ }^{1}$ Currently, less than $50 \%$ of major depressive disorder (MDD) patients achieve remission following treatment with antidepressants. There is an urgent need to understand what factors determine who is likely to benefit, and who is not, from commonly used first-line treatments. Childhood trauma, particularly due to abuse and neglect, is among the most significant contributors to the subsequent development of MDD. ${ }^{2-7}$ Despite this evidence, the rates of childhood trauma in outpatient depressed populations have not been well established and it remains unknown whether trauma predicts treatment outcomes in these outpatient populations. Thus, our aims were to establish the rates of trauma, overall and for individual types of trauma, in a large global sample of depressed outpatients and to assess whether exposure to these traumatic events is a general and/or differential predictor of outcomes following treatment with one of three commonly used antidepressants.

The exposure to significant traumatic stressors in childhood, including physical and sexual abuse and loss of parents, has been associated with an increased risk of depression. ${ }^{3,5}$ Exposure to early trauma and abuse increase the likelihood of meeting criteria for a major depressive episode at any point in life, ${ }^{6}$ compared with the absence of exposure to trauma. Childhood abuse predicts poorer outcomes, including earlier-onset depression, a higher risk of recurrent depression, more severe course of illness and greater chronicity. 2,8

Several studies suggest that a history of pre-pubertal abuse or trauma also moderates response to treatment, however, with variable results. ${ }^{8}$ Nemeroff et al. ${ }^{9}$ studied 681 patients with chronic depression and their response to an antidepressant (nefazodone) or CBASP (cognitive behavioral analysis of psychotherapy), a form of cognitive behavioral therapy (CBT). Patients without a history of

\footnotetext{
${ }^{1}$ Department of Psychiatry and Behavioral Sciences, Stanford University School of Medicine, Stanford, CA, USA; ${ }^{2}$ Sierra-Pacific Mental Illness Research, Education, and Clinical Center, Veterans Affairs Palo Alto Health Care System, Palo Alto, CA, USA; ${ }^{3}$ Department of Psychiatry, The Ohio State University College of Medicine, Columbus, OH, USA and

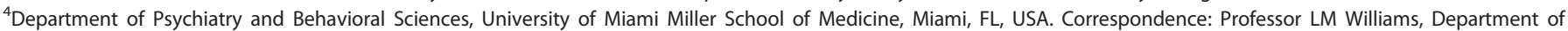
Psychiatry and Behavioral Sciences, Stanford University School of Medicine, 401 Quarry Road Stanford, Stanford, CA 94305, USA.

E-mail: leawilliams@stanford.edu

${ }^{5}$ These two authors contributed equally to this work.

Received 14 April 2015; revised 30 December 2015; accepted 5 March 2016
} 
pre-pubertal stressor had an equivalent response to psychotherapy and nefazodone, and the best response to the combination treatment. ${ }^{10}$ However, patients who reported a history of parental loss before the age of 15 , parental neglect or childhood physical or sexual abuse were significantly less likely to respond to pharmacotherapy alone. Furthermore, combination treatment did not substantially improve the outcome versus CBASP alone.

Some studies have suggested that specific types of abuse rather than a general history of trauma moderates treatment response. In the Treatment of Resistant Depression in Adolescents Study, 334 (ref. 11) adolescents who did not respond to a selective serotoninreuptake inhibitor were randomized to receive 12 weeks of an alternative selective serotonin-reuptake inhibitor alone, or combined with $\mathrm{CBT}$, or venlafaxine alone (a serotonin-norepinephrine reuptake inhibitor; SNRI) or with CBT. Those without a history of abuse did better on the combination treatment than on antidepressant monotherapy (62.8 vs $37.0 \%$ ). Those with a history of sexual abuse responded equally to monotherapy and combination treatment ( 48.3 vs $42.3 \%$ ), whereas those with a history of physical abuse responded substantially better to antidepressant monotherapy than combination treatment (52.4 vs $18.4 \%)$.

The Treatment for Adolescents with Depression Study ${ }^{12}$ compared the efficacy of 12 weeks fluoxetine, CBT, their combination and placebo in 427 adolescents with MDD, divided into four groups by their history: (1) no trauma; (2) trauma, no abuse; (3) physical abuse; and (4) sexual abuse. The no-trauma group responded to fluoxetine, with no effect of CBT over placebo. For teens with a history of trauma or physical abuse, no treatment was more effective than placebo. In the sexual abuse group, placebo appeared more effective than CBT. These findings are in contrast to Nemeroff et al., ${ }^{9}$ in which psychotherapy appeared to be more effective than medication in depressed adults with a history of trauma or abuse. The difference in participant population (chronically depressed and adult vs children) could explain the discrepancy. Pediatric studies have generally suggested that a history of childhood trauma predicted a poorer response to psychotherapy. ${ }^{13-15}$ A recent meta-analysis ${ }^{8}$ concluded that a history of early-life stress predicted poorer response to pharmacotherapy and combination pharmacotherapy/psychotherapy, and a trend for poor response to psychotherapy as well.

The international Study to Predict Optimized Treatment in Depression (iSPOT-D) enrolled nonpsychotic MDD patients into a real-world practical effectiveness trial, primarily to identify pretreatment characteristics as general or differential predictors of response to antidepressants that could change how practitioners select among antidepressant medications. ${ }^{16}$

In this study, we report on the effects of early-childhood trauma as a predictor of acute antidepressant treatment outcomes at 8 weeks post treatment in the 1008 depressed participants from iSPOT-D. Our aims were to test whether (i) the prevalence of earlylife trauma differs in iSPOT-D MDD participants compared with matched healthy controls; (ii) the overall amount of 'load' of earlylife trauma is a general predictor of acute antidepressant treatment outcomes defined by response, remission and change in symptom severity; (iii) exposure to a specific type of abuse is a specific predictor of acute antidepressant treatment outcomes; and (iv) overall 'load' of early-life trauma and exposure to a specific type of abuse are differential predictors of acute antidepressant treatment outcomes for different types of antidepressants.

\section{MATERIALS AND METHODS}

Sites and practitioners

The iSPOT-D is a multi-site, randomized practical clinical trial ${ }^{9}$ conducted at 17 sites in the United States, the Netherlands, Australia, New Zealand and South Africa. These sites include eight academic and nine private clinical settings. We refer to these as the 'study management' sites because they manage recruitment and assessments. Most also act as the hub for a broader network of 'care delivery' providers at community general practices and university general health centers, which prescribe and manage medication following usual clinical care.

\section{Design}

The iSPOT-D is a multiple-phase, multi-site, real-world effectiveness study. The primary outcome is response at 8 weeks post baseline. ${ }^{16}$ The first phase enrolled 1008 MDD participants and 336 matched controls. The participants were assessed at the study sites at baseline and 8 weeks later.

\section{Participants}

The study enrolled 1008 adults (aged 18-65 years) with a diagnosis of current, single-episode or recurrent nonpsychotic MDD and 336 controls with no diagnosis of mental disorder. Each site enrolled both MDD patients and healthy controls. The minimum number of participants per site was 30 and the maximum 200. The participants were enrolled between December 2008 and January 2012.

Psychiatric inclusion and exclusion criteria for MDD and controls were based on demographic information, medical and medication history, the Mini International Neuropsychiatric Interview, the 17-item Hamilton Rating Scale for Depression $\left(\mathrm{HRSD}_{17}\right)^{12}$ to assess depressive severity (score $\geqslant 16$ for inclusion) and a urine toxicology screen. The exclusion criteria for all the participants included current or past diagnosis of psychosis, bipolar disorder, eating disorder, posttraumatic stress disorder and obsessive compulsive disorder as well as Axis II personality disorder. Known medical disease that might interfere with assessments was also exclusionary. In addition, for the control group, current or past history of MDD was exclusionary and for the MDD group, participants needed to meet inclusion criteria for MDD without psychosis (see Williams et al. ${ }^{16}$ for study design and Saveanu et al. ${ }^{17}$ for complete list of inclusion/exclusions). The medical history and adverse events for 1 year following the first visit both for controls and MDD participants were also recorded. The study was approved by each site's institutional review board and was carried out in accordance with the Declaration of Helsinki. All the participants provided written informed consent and received a stipend for their time and travel.

The participants with MDD had moderate-to-severe depression, with an average $\mathrm{HRSD}_{17}$ score of $21.9 \pm 4.1$ (corresponding 16-item Quick Inventory of Depressive Symptomatology-Self-Rated (QIDS-SR ${ }_{16}$ ) score of $14.5 \pm 3.8$ ). Almost $90 \%$ of the participants had recurrent MDD (average: five episodes). The average age at first episode was $22.9 \pm 12.0$ years.

The sample size and power were determined as part of the protocol development. ${ }^{16}$ Our sample size was designed to provide statistical power of at least $89 \%$ power to detect small effects for predictors (odds ratio (OR) of 1.3 per standard deviation change in extent of trauma at the pretreatment baseline) at an alpha level of $P<0.05$ and $94 \%$ power to detect medium effects (OR of 1.5). Further details about the participant sample are reported in Saveanu et al. ${ }^{17}$

\section{Study treatment}

After pre-treatment assessments, MDD participants were randomized to receive escitalopram, sertraline or venlafaxine-extended release (XR). As outlined in the protocol for iSPOT-D, ${ }^{16}$ these three antidepressants were chosen because they are commonly used in practice in each country in which participating sites are located, are approved for MDD in the country of each participating site and have distinct pharmacological properties, which may enable the identification of moderators. The antidepressants were prescribed and doses adjusted by the participant's treating clinician according to routine clinical practice. Equal numbers of participants were randomly assigned to each treatment. The average treatment duration was $6.1 \pm 2.8$ weeks at the time of follow-up assessment.

The average daily dose for each antidepressant was consistent with usual clinical prescribing practices (escitalopram: $12.3 \mathrm{mg}$ per day; sertraline: $61.1 \mathrm{mg}$ per day; venlafaxine-XR: $83.4 \mathrm{mg}$ per day). Doses were in the lower end of recommended ranges compared with typical trials, consistent with the practical design of the trial which mirrored practice in primary care and community settings. Only $8 \%$ of participants used concomitant psychotropic medications, mostly anxiolytics (3.8\%) and sleep aids (1.2\%).

Adverse events were reported by 452 participants (44.8\%), 88.3\% likely related to the antidepressants. Six serious adverse events involved hospitalization, two for suicidal ideation/attempt, and led to early 


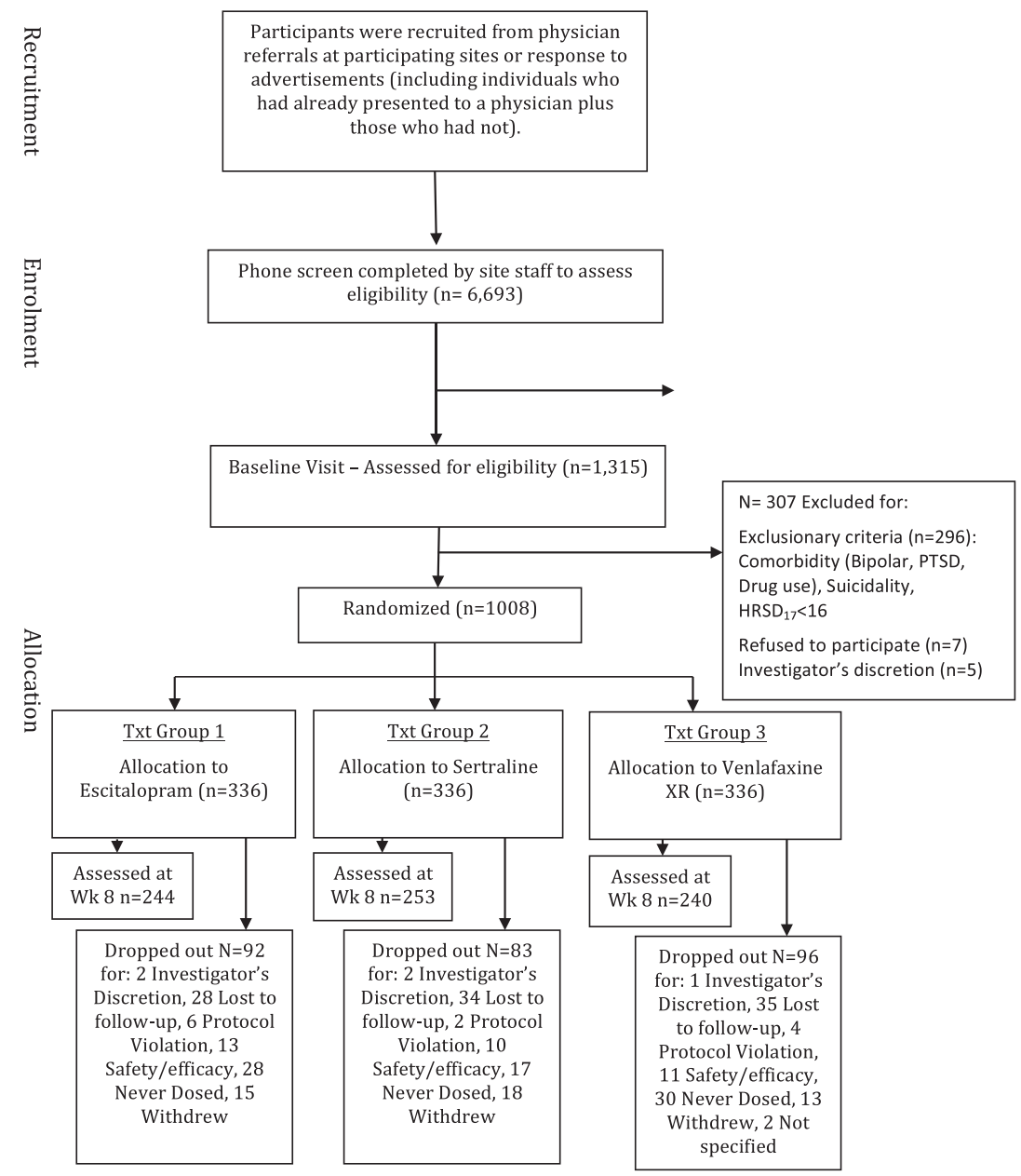

Figure 1. CONSORT chart. HRSD 17 , 17-item Hamilton Rating Scale for Depression; PTSD, posttraumatic stress disorder.

withdrawal. The adverse event rates did not significantly differ across treatment arms. ${ }^{17}$

\section{Treatment outcomes}

The primary outcome was rate of response $\left(\geqslant 50 \%\right.$ decrease in $\left[\mathrm{HRSD}_{17}\right]$ scores from baseline to week 8$)$ and remission $\left(\mathrm{HRSD}_{17}\right.$ score $\leqslant 7$ ) to treatment. Secondary outcomes included response and remission by the QIDS-SR $_{16}$ (response: $\geqslant 50 \%$ decrease from baseline, remission: score $\leqslant 5) .{ }^{17,18}$ By the $\mathrm{HRSD}_{17}, 62.2 \%$ of participants met the criteria for response at week 8 , and $45.4 \%$ were in remission. By the QIDS-SR $16,53.3 \%$ of participants responded and $37.6 \%$ were in remission (Saveanu et al. ${ }^{17}$ ). The response and remission rates did not significantly differ between the three treatment groups. We also assessed change in symptom severity on the HRSD $_{17}$ and the QIDS-SR 16 as a dimensional measure.

There was a $28.4 \%$ attrition rate that represented participants who were not medication adherent at week 8 or who withdrew from the study before week 8 (Figure 1).

\section{Assessment of childhood trauma}

We used the Early-Life Stress Questionnaire (ELSQ) ${ }^{19}$ to assess the role of childhood trauma in relation to antidepressant outcomes. The ELSQ comprises 18 items, which assess exposure to specific traumatic events in the first 17 years of life (Table 1) and which are equivalent to the trauma items assessed by the Child Abuse and Trauma Scale. ${ }^{20}$ These events represent previously identified categories of trauma, including interpersonal violation (physical, psychological and sexual abuse, neglect, domestic violence, bullying), family breakup (divorce, separation, conflict), family health (death, life-threatening illness), personal health (hospitalization, lifethreatening illness or injury), disaster/war (natural disaster, war), birth complications or adoption. ${ }^{20-23}$ Each item is scored dichotomously for the presence/absence of exposure to each type of trauma. For each type of trauma endorsed as 'present', participants also reported the age range in which the trauma occurred or first occurred $(0-3,4-7,8-12$ or $13-17$ years of age). Previously, reports of childhood trauma using the ELSQ have been shown to correspond to registry-reported prevalence, and that reported trauma is predictive of severity of depressive symptoms. ${ }^{19}$

Extent of exposure to traumatic events was not found to differ across site (chi-square $=10.954, \mathrm{df}=6, P=0.090$ ) or across country (chi-square $=$ $6.134, \mathrm{df}=4, P=0.189$ ).

\section{Statistical analysis}

All analyses were conducted for the intention-to-treat sample. We established response and remission status based on the patients who completed treatment and the 8-week follow-up assessment (Figure 1). Responses to the ELSQ items were treated as categorical variables.

1. To test the hypothesis that prevalence of early-life trauma differs in MDD patients compared with controls, we used chi-square analysis and compared groups on each type of trauma.

2. We used logistic regression models to test whether overall trauma is a general predictor of acute treatment outcomes for response and remission defined by the $\mathrm{HRSD}_{17}$ and $\mathrm{QIDS} \mathrm{SR}_{16}$. Linear regression models were used to test prediction of dimensionally defined severity of symptoms at week 8 , controlling for pre-treatment baseline severity.

3. We also used logistic and linear regression models to test whether abuse is a specific predictor of treatment outcomes. Given the focus on a particular trauma category, we used a corrected alpha level of 0.01 .

4. We used logistic and linear regression models and included an interaction term for trauma by type of antidepressant to test whether 
Table 1. Prevalence of early-childhood trauma in depressed patients and in healthy controls, according to the category and type of trauma

\begin{tabular}{|c|c|c|c|c|}
\hline \multirow[t]{2}{*}{ Category and type of trauma (ELSQ items: yes/no questions) } & \multicolumn{2}{|c|}{$\%$ Prevalence of trauma } & \multicolumn{2}{|c|}{ Group difference } \\
\hline & $\begin{array}{c}M D D \\
\mathrm{~N}=1008 \\
\mathrm{n}(\%)\end{array}$ & $\begin{array}{c}\text { Control } \\
\mathrm{N}=336 \\
\mathrm{n}(\%)\end{array}$ & $\chi^{2}$ & P-value \\
\hline \multicolumn{5}{|l|}{ Abuse/interpersonal violation } \\
\hline Were you physically abused? & $219(21.7)$ & $18(5.4)$ & 41.925 & $<0.001$ \\
\hline Were you sexually abused? & $164(16.3)$ & $18(5.4)$ & 27.758 & $<0.001$ \\
\hline Did you witness domestic violence within your family? & $326(32.3)$ & 49 (14.6) & 36.560 & $<0.001$ \\
\hline Did you experience sustained bullying or rejection by schoolmates? & $452(44.8)$ & $60(17.9)$ & 82.231 & $<0.001$ \\
\hline \multicolumn{5}{|l|}{ Family breakup } \\
\hline Did your parents divorce or separate? & $408(40.5)$ & $103(30.7)$ & 11.894 & 0.02 \\
\hline Were you separated for a long period from a parent, brother or sister? & 439 (43.6) & $61(18.2)$ & 30.035 & $<0.001$ \\
\hline Was there sustained conflict within your family? & $516(51.2)$ & $112(33.3)$ & 70.875 & $<0.001$ \\
\hline \multicolumn{5}{|l|}{ Family health/death } \\
\hline \multicolumn{5}{|l|}{ Personal health } \\
\hline Did you undergo major surgery or repeated hospitalization? & $168(16.7)$ & $32(9.5)$ & 9.559 & 0.05 \\
\hline Did you experience a life-threatening illness or injury? & $100(9.9)$ & $27(8.0)$ & 5.227 & 0.27 \\
\hline \multicolumn{5}{|l|}{ Disaster/war } \\
\hline Did you witness first-hand a natural disaster such as earthquake, flood or fire? & $121(12.0)$ & $39(11.6)$ & 4.229 & 0.38 \\
\hline Did you witness warfare? & $29(2.9)$ & $7(2.1)$ & 0.357 & 0.55 \\
\hline \multicolumn{5}{|l|}{ Other traumatic events } \\
\hline Were you born prematurely, or experience other birth complications? & $132(13.1)$ & $29(8.6)$ & 3.895 & 0.048 \\
\hline Were you adopted? & $43(4.3)$ & $8(2.4)$ & 3.399 & 0.49 \\
\hline Was your house destroyed by fire or other means? & $43(4.3)$ & $14(4.2)$ & 2.711 & 0.61 \\
\hline
\end{tabular}

overall trauma load or specific exposure to childhood abuse is a differential predictor of treatment outcomes for different types of antidepressants (escitalopram, sertraline, venlafaxine-XR).

In each regression model, clinical site, age and baseline level of

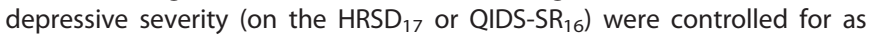
covariates.

On the basis of the exponential beta values in the regression models, we generated the OR for each significant effect. The OR for interaction terms reflects how much greater/lower the likelihood of response/remission is in a multiplicative sense for treatment type.

\section{RESULTS}

Prevalence of early-life trauma

To confirm the absence of mental disorder meeting criteria for DSM-IV, healthy controls were assessed by a MINI structured interview. They also completed all the assessments and had the same visit schedule as MDD participants. MDD participants were exposed to significantly more early-life stress than the healthy controls (Table 1): $62.5 \%$ of MDD participants reported more than two traumatic events compared with $28.4 \%$ of controls (Table 1 ). Most notably, MDD participants had a fourfold or higher rate of exposure to abuse (sexual, physical and emotional) than healthy controls (Table 1). Other types of interpersonal violation (neglect, witnessing domestic violence and bullying or rejection) were also significantly increased. More than $40 \%$ of the MDD participants reported experience of emotional abuse or bullying/rejection at school compared with less than $10 \%$ and $20 \%$, respectively for controls. Having experienced parent divorce, separation from family, conflict with family or undergone major surgery or hospitalizations was also more frequent among MDD participants than controls, whereas other potential traumatic events like death in the family or natural disasters were not. Supplementary analyses showed that the group difference in ELSQ scores did not differ as a function of gender; hence, gender was not included as an additional variable in subsequent analyses.

Overall presence of early-life trauma as a predictor of response and remission

Of 1008 participants randomized to treatment, 286 dropped out and 722 were assessed at week 8 (Figure 1). Overall presence of at least one early-life traumatic event was not a significant predictor of the percentage change in symptom severity from pre- to posttreatment, assessed by the $\mathrm{HRSD}_{17}$ and QIDS-SR ${ }_{16}$. Overall trauma was also not a significant predictor of response or remission defined categorically by the HRSD 17 and QIDS-SR ${ }_{16}$.

Specific contribution of abuse as a predictor of response and remission

The experience of abuse was a significant predictor of both $\mathrm{HRSD}_{17}$-defined remission (chi-square $=49.782, \mathrm{df}=12, P<0.0001$ ) and response (chi-square $=72.769, \mathrm{df}=12, \quad P<0.0001$ ). The experience of abuse at the age of 4 to 7 years contributed specifically and significantly to prediction for both $\mathrm{HRSD}_{17}$-defined response $(P=0.034 ; \quad \mathrm{OR}=1.574)$ and remission $(P=0.032$; $\mathrm{OR}=1.606$ ), indicating that participants were about 1.6 times less likely to achieve response or remission if exposed to abuse at this age (Figure 2). 
a

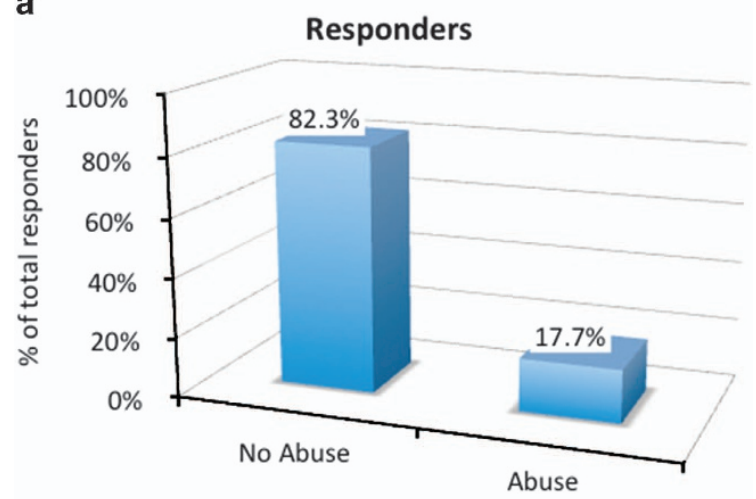

b

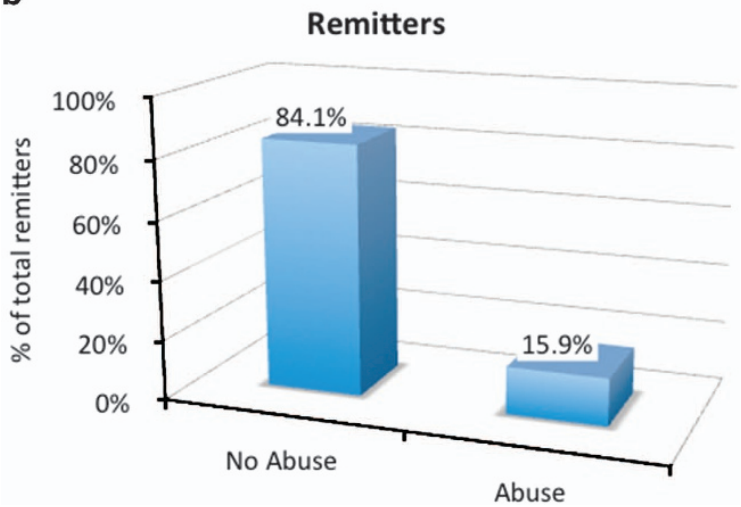

Figure 2. The effect of abuse at age 4-7 years on prediction for $\mathrm{HRSD}_{17}$ response and remission. HRSD 17 , 17-item Hamilton Rating Scale for Depression.

Abuse occurring at age 4 to 7 years was also a predictor of percentage change in clinician-rated symptom severity assessed by the $\mathrm{HRSD}_{17}(\mathrm{~F}=2.787, P=0.007$; specific contribution of abuse at 4 to 7 years; $t=2.573, P=0.01 ; 50 \%$ mean symptom improvement in patients exposed to abuse at 4 to 7 years versus $58 \%$ in those not exposed). Similarly, abuse occurring at age 4 to 7 years was a predictor of dimensional change in self-reported symptom severity assessed by the QIDS-SR $16 \quad(F=6.507$, $P<0.0001$; specific contribution of abuse at 4 to 7 years; $t=2.242, P=0.025 ; 40 \%$ mean symptom improvement in patients exposed to abuse at 4 to 7 years versus $47 \%$ in those not exposed).

Early-life abuse as a predictor of response and remission for specific types of treatment

Overall, the presence of early-life stressors occurring before 18 years of age did not interact with the type of treatment to predict response or remission. However, exposure to the specific trauma of abuse did interact with types of treatment to predict response and remission. Abuse included physical, sexual and emotional abuse items, which have been shown to form a cohesive factor. ${ }^{19}$

The experience of abuse predicted $\mathrm{HRSD}_{17}$-defined response according to type of treatment (omnibus chi-square $=49.421$, $\mathrm{df}=13, P<0.0001)$, specifically due to abuse at 4 to 7 years of age $(P=0.043$, interaction $\mathrm{OR}=1.225)$. The experience of abuse also predicted $\mathrm{HRSD}_{17}$-defined remission according to type of treatment (omnibus chi-square $=75.136, \quad \mathrm{df}=13, \quad P<0.0001$ ), specifically due to abuse at 4 to 7 years of age $(P=0.033$, interaction $O R=1.251$ ). The experience of abuse also predicted QIDS-SR $_{16}$-defined remission according to type of treatment (omnibus chi-square $=36.322, \mathrm{df}=13, \quad P<0.0001$ ), specifically due to abuse at 4 to 7 years of age $(P=0.049$, interaction $\mathrm{OR}=1.260$ ). For sertraline, in particular, participants who were abused at this age showed significantly less improvement in both clinician $(P<0.0001)$ and self-rated $(P=0.007)$ symptoms, than with escitalopram and venlafaxine-XR treatments.

\section{DISCUSSION}

The iSPOT-D is the first real-world practical trial to assess the prevalence of childhood trauma and its impact on antidepressant outcomes in an international sample of outpatient treatment seekers with MDD. These outpatients were found to have a fourfold higher incidence of childhood abuse than their healthy peers, and a twofold higher incidence of early exposure to other traumatic events. The greater the exposure to abuse in particular, the less likely these depressed patients were to remit following treatment with one of the three commonly prescribed antidepressants. Our results suggest that remission rates may be especially low when abuse occurs during the very early period of 4-7 years of age, and following treatment with sertraline.

Overall, the higher rate of trauma observed in the present MDD sample is in line with registry and observational studies. ${ }^{4,9}$ Thus, childhood trauma and especially abuse may contribute to the development of depression observed in routine practice in multiple outpatient settings spanning primary and specialist care settings. Our findings suggest that abuse in particular, and not overall exposure to traumatic events, predicts a lower rate of acute response and remission after antidepressant therapy. Sexual, physical and emotional abuse compared with other types of trauma (such as death of a parent/sibling, personal lifethreatening illness/injury, or disaster), may have a specific impact on the neurobiological mechanisms of non-response to treatment. Neuroimaging studies suggest that there may be a differential effect of childhood sexual abuse on the subsequent functioning of emotional brain circuits in adulthood depression. ${ }^{24}$ Childhood abuse has also been associated with a greater sensitivity to stress, ${ }^{4}$ cognitive impairment, ${ }^{25}$ alterations in brain morphometry ${ }^{26-30}$ and immune and metabolic abnormalities ${ }^{31}$ that may impact the course of depression and capacity to respond to antidepressants. It is also possible that abuse may recur and that it is the recurrence of the trauma that produces poor treatment outcomes.

In addition to the type of stressor, our results suggest that there is a critical period (4 to 7 years) in which childhood trauma occurs and has the most significant impact on subsequent poor response to antidepressants in adulthood. There is a rapidly growing body of work to suggest that gene polymorphisms and epigenetic modifications interact with childhood trauma to exert their effect on risk for depression, ${ }^{32-34}$ and that this effect is greatest at critical neurodevelopmental periods. ${ }^{7,35}$ This evidence is consistent with the view that the extent of brain plasticity varies during development, such that trauma occurring during critical neurodevelopmental periods may alter brain morphometry, circuit function, endocrine regulation, immune function and subsequent physiologic reactions to stress in an enduring way. ${ }^{28,29,36,37}$

Abuse occurring at age 4 to 7 years was associated with significantly poorer outcomes following the treatment with sertraline compared with the other selective serotonin-reuptake inhibitor escitalopram and the SNRI venlafaxine-XR. The participants who were abused at this age showed significantly less improvement in both clinician and self-rated symptoms following 8 weeks treatment with sertraline. Sertraline, in contrast to other serotonin-reuptake antidepressants, has an additional relatively specific effect on inhibiting dopamine. ${ }^{38}$ There is some evidence that subgroups of patients are also characterized by dopamine circuit dysfunction ${ }^{39}$ and number of traumatic events has been associated with higher ventral striatal dopamine response to amphetamine. ${ }^{40}$ Although speculative, these lines of evidence 
suggest that a possible dopamine-related mechanism might contribute to our specific observation of especially poor outcomes following sertraline in those exposed to early abuse.

\section{Clinical translational significance}

Here, we provide evidence from a well-powered study that outpatients with MDD have a fourfold higher incidence of childhood abuse than their healthy peers, and a twofold higher incidence of early exposure to other traumatic events. The greater the exposure to trauma, the less likely these depressed patients were to remit following antidepressant response. Thus, the translational significance of these findings is that in routine clinical management of depression it may be important to screen for childhood trauma to identify those patients that may not benefit from standard first-line antidepressants and may require additional therapy to more directly address the impact of trauma.

\section{Limitations}

The results should be interpreted within the context of several potential limitations of the study. First, there may be limitations due to sampling. We had a higher percentage of males relative to females than reported in previous studies such as STAR*D. This difference might be because we used a practical biomarker trial design and that males were more likely to report depression and trauma in this context. However, because women have previously been more likely to report trauma than men, ${ }^{41}$ future studies are required to establish the generalizability of our findings.

The study might also be limited due to the assessment of earlychildhood trauma by the ELSQ. The scale is dependent on the recollection of the subject, and age 4-7 years may be the earliest reliable recollection rather than representing only a specific window of vulnerability. However, this limitation would not account for the lack of specific effect of abuse experienced after 7 years of age. Retrospective reports of childhood trauma, such as those assessed by the ELSQ, may have some bias but also tend to err on the side of false negatives rather than false positives. ${ }^{42}$ The prevalence of trauma reported retrospectively using the ELSQ is comparable with prevalence rates from archival records ${ }^{19}$ suggesting that such retrospective reports are also reliable. Nonetheless, it would be important to establish the reproducibility of our findings using additional and independent measures. Design limitations of this study include the reliance on only three first-step antidepressants, though they are commonly used in practice. Doses were lower than midrange of the recommended range, perhaps because the response and remission rates were large enough that further dose escalation was not warranted in many patients. Furthermore, as dose ranges were reflective of usual management practices and since primary care physicians prescribe about two-thirds of antidepressants, ${ }^{43}$ these ranges are an appropriate starting point for identifying predictors of outcome in real-world management settings. Although the present sample is well powered, we recognize that the findings are limited to this sample and that it would be beneficial to seek to replicate the findings in another sample. The iSPOT-D is designed to address future replication in a second cohort of a similar sample size.

\section{CONCLUSION}

Here we provide a systematic investigation to show that there is a fourfold increase in the rate of childhood trauma, especially physical, emotional and sexual abuse, ${ }^{35}$ in outpatients with depression. Abuse occurring before the age of 7 years predicts substantially poorer response and remission outcomes for commonly prescribed antidepressants. These outcomes may be poorest for sertraline. These results suggest that it is important to assess for childhood trauma in the outpatient management of depression, and to consider alternative or supplemental treatments for patients with a trauma history.

\section{CONFLICT OF INTEREST}

The iSPOT-D sponsor central management team managed the operations of the sites and the data storage and quantification. Interpretation of the data was undertaken independently from the study sponsor and preparation and review of the manuscript was undertaken by the academic authors. LMW has received consultant fees from Brain Resource and from Humana. CD has received research funding from Brain Resource, St. Jude, Jannsen, Assurex, Roche, Takeda and has received consultant fees from Pfizer and Genentech. A-MD has received research funding from Brain Resource. AFS has served as a consultant to Bay City Capital, BrainCells, CeNeRx, Cervel, Depomed, Eli Lilly, Forum, Genentech, Gilead, Jazz, Lundbeck/Takeda, McKinsey, Merck, MSI, Neuronetics, Novadel, One-Carbon, PharmaNeuroBoost, Sunovion, Synosia and Xhale; he has received honoraria from Merck; he has equity in Amnestix, BrainCells, CeNeRx, Corcept (co-founder), Delpor, Forest, Merck, Neurocrine, Novadel, Pfizer, PharmaNeuroBoost, Somaxon, Synosis, Titan and Xhale; and he receives royalties from Stanford University for patents on mifepristone use and the pharmacogenetics of antidepressant response. In the last 3 years, CBN has received a Scientific Advisory Board/Board of Directors fee from PharmaNeuroboost, Clintara/Bracket, Xhale, Taisho, Fortress, Takeda, Janssen/Ortho-McNeil and George West Mental Health Foundation. He holds stock or stock options in Xhale, Clintara/ Bracket, Seattle Genetics, Celgene, Titan and Network Life Sciences. CBN has patents for Methods and devices for the transdermal delivery of lithium (US $6375990 \mathrm{BI}$ ) and Method to estimate serotonin and norepinephrine transporter occupancy after drug treatment using patient or animal serum (provisional filing April 2001). LMW was the overall academic PI for iSPOT-D (2008-2013), CD is the PI for the Stanford iSPOT-D site, A-MD is co-I for the Ohio State iSPOT-D site, AFS is co-I for the Stanford iSPOT-D site and CBN is Co-I for the Miami University iSPOT-D site. Analyses were undertaken by $L M W, C D$ and $C B N$ and all the authors participated in interpreting results and drafting the manuscript. CD and LMW had full access to all the data in the study and they take responsibility for the integrity of the data and the accuracy of the data analysis.

\section{ACKNOWLEDGMENTS}

We acknowledge the iSPOT-D Investigators Group, the contributions of principal and co-investigators at each site and Claire Day, Brain Resource global coordinator for iSPOT-D (2008-2015) and the Scoring Server management by Donna Palmer (Brain Resource). We gratefully acknowledge the editorial support of Jon Kilner (Pittsburgh, PA, USA). The iSPOT-D is sponsored by Brain Resource Company Operations.

\section{REFERENCES}

1 Whiteford HA, Degenhardt L, Rehm J, Baxter AJ, Ferrari AJ, Erskine HE et al. Global burden of disease attributable to mental and substance use disorders: findings from the Global Burden of Disease Study 2010. Lancet 2013; 382: 1575-1586.

2 Bernet CZ, Stein MB. Relationship of childhood maltreatment to the onset and course of major depression in adulthood. Depress Anxiety 1999; 9: 169-174.

3 Dube SR, Anda RF, Whitfield CL, Brown DW, Felitti VJ, Dong M et al. Long-term consequences of childhood sexual abuse by gender of victim. Am J Prev Med 2005; 28: 430-438.

4 Hammen C, Henry R, Daley SE. Depression and sensitization to stressors among young women as a function of childhood adversity. J Consult Clin Psychol 2000; 68: 782-787.

5 Kendler KS, Kessler RC, Walters EE, MacLean C, Neale MC, Heath AC et al. Stressful life events, genetic liability, and onset of an episode of major depression in women. Am J Psychiatry 1995; 152: 833-842.

6 Kessler RC, Davis CG, Kendler KS. Childhood adversity and adult psychiatric disorder in the US National Comorbidity Survey. Psychol Med 1997; 27: 1101-1119.

7 Nemeroff CB, Binder E. The preeminent role of childhood abuse and neglect in vulnerability to major psychiatric disorders: toward elucidating the underlying neurobiological mechanisms. J Am Acad Child Adolesc Psychiatry 2014; 53: 395-397.

8 Nanni V, Uher R, Danese A. Childhood maltreatment predicts unfavorable course of illness and treatment outcome in depression: a meta-analysis. Am J Psychiatry 2012; 169: 141-151.

9 Nemeroff CB, Heim CM, Thase ME, Klein DN, Rush AJ, Schatzberg AF et al. Differential responses to psychotherapy versus pharmacotherapy in patients with chronic forms of major depression and childhood trauma. Proc Natl Acad Sci USA 2003; 100: 14293-14296. 
10 Keller MB, McCullough JP, Klein DN, Arnow B, Dunner DL, Gelenberg AJ et al. A comparison of nefazodone, the cognitive behavioral-analysis system of psychotherapy, and their combination for the treatment of chronic depression. $N$ Engl J Med 2000; 342: 1462-1470.

11 Shamseddeen W, Asarnow JR, Clarke G, Vitiello B, Wagner KD, Birmaher B et al. Impact of physical and sexual abuse on treatment response in the Treatment of Resistant Depression in Adolescent Study (TORDIA). J Am Acad Child Adolesc Psychiatry 2011; 50: 293-301.

12 Lewis CC, Simons AD, Nguyen L, Murakami JL, Reid MW, Silva SG et al. Impact of childhood trauma on treatment outcome in the Treatment for Adolescents with Depression Study (TADS). J Am Acad Child Adolesc Psychiatry 2010; 49: 132-140.

13 Asarnow JR, Emslie G, Clarke G, Wagner KD, Spirito A, Vitiello B et al. Treatment of selective serotonin reuptake inhibitor-resistant depression in adolescents: predictors and moderators of treatment response. J Am Acad Child Adolesc Psychiatry 2009; 48: 330-339.

14 Barbe RP, Bridge JA, Birmaher B, Kolko DJ, Brent DA. Lifetime history of sexual abuse, clinical presentation, and outcome in a clinical trial for adolescent depression. J Clin Psychiatry 2004; 65: 77-83.

15 Shirk SR, Karver M. Prediction of treatment outcome from relationship variables in child and adolescent therapy: a meta-analytic review. J Consult Clin Psychol 2003; 71: 452-464.

16 Williams LM, Rush AJ, Koslow SH et al. International Study to Predict Optimized Treatment for Depression (iSPOT-D), a randomized clinical trial: rationale and protocol. Trials 2011; 12: 4.

17 Saveanu R, Etkin A, Duchemin A-E, Goldstein-Piekarski A, Gyurak A, Debattista C et al. The International Study to Predict Optimized Treatment in Depression (iSPOT-D): outcomes from the acute phase of antidepressant treatment. J Psychiatric Res 2015; 61: 1-12.

18 Rush AJ, Trivedi MH, Ibrahim HM, Carmody TJ, Arnow B, Klein DN et al. The 16-Item Quick Inventory of Depressive Symptomatology (QIDS), clinician rating (QIDS-C), and self-report (QIDS-SR): a psychometric evaluation in patients with chronic major depression. Biol Psychiatry 2003; 54: 573-583.

19 Trivedi MH, Rush AJ, Ibrahim HM, Carmody TJ, Biggs MM, Suppes T et al. The Inventory of Depressive Symptomatology, Clinician Rating (IDS-C) and Self-Report (IDS-SR), and the Quick Inventory of Depressive Symptomatology, Clinician Rating (QIDS-C) and Self-Report (QIDS-SR) in public sector patients with mood disorders: a psychometric evaluation. Psychol Med 2004; 34: 73-82.

20 Chu DA, Williams LM, Harris AW, Bryant RA, Gatt JM. Early life trauma predicts self-reported levels of depressive and anxiety symptoms in nonclinical community adults: Relative contributions of early life stressor types and adult trauma exposure. J Psychiatr Res 2013; 47: 23-32.

21 Sanders B, Becker-Lausen E. The measurement of psychological maltreatment: early data on the Child Abuse and Trauma Scale. Child Abuse Negl 1995; 19: 315-323.

22 Becker-Lausen E, Sanders B, Chinsky JM. Mediation of abusive childhood experiences: depression, dissociation, and negative life outcomes. Am J Orthopsychiatry 1995; 65: 560-573.

23 Kent A, Waller G. The impact of childhood emotional abuse: an extension of the Child Abuse and Trauma Scale. Child Abuse Negl 1998; 22: 393-399.

24 Mark TL, Levit KR, Buck JA. Psychotropic drug prescriptions by medical specialty. Psychiatr Serv 2009; 60: 1167.

25 Majer M, Nater UM, Lin JM, Capuron L, Reeves WC. Association of childhood trauma with cognitive function in healthy adults: a pilot study. BMC Neurol 2010; 10: 61.

26 Heim C, Shugart M, Craighead WE, Nemeroff CB. Neurobiological and psychiatric consequences of child abuse and neglect. Dev Psychobiol 2010; 52: 671-690.
27 Heim CM, Mayberg HS, Mletzko T, Nemeroff CB, Pruessner JC. Decreased cortical representation of genital somatosensory field after childhood sexual abuse. Am $J$ Psychiatry 2013; 170: 616-623.

28 Huang LT. Early-life stress impacts the developing hippocampus and primes seizure occurrence: cellular, molecular, and epigenetic mechanisms. Front $\mathrm{Mol}$ Neurosci 2014; 7: 8.

29 Lupien SJ, McEwen BS, Gunnar MR, Heim C. Effects of stress throughout the lifespan on the brain, behaviour and cognition. Nat Rev Neurosci J 2009; 10: 434-445.

30 McCrory E, De Brito SA, Viding E. The link between child abuse and psychopathology: a review of neurobiological and genetic research. $J$ R Soc Med 2012; 105: 151-156.

31 Danese A, Moffitt TE, Harrington $H$, Milne BJ, Polanczyk G, Pariante CM et al. Adverse childhood experiences and adult risk factors for age-related disease: depression, inflammation, and clustering of metabolic risk markers. Arch Pediatr Adolesc Med 2009; 163: 1135-1143.

32 Skokauskas N, Carballedo A, Fagan A, Frodl T. The role of sexual abuse on functional neuroimaging markers associated with major depressive disorder. World $J$ Biol Psychiatry 2015; 16: 513-520.

33 Gatt JM, Nemeroff CB, Schofield PR, Paul RH, Clark CR, Gordon E et al. Early life stress combined with serotonin $3 \mathrm{~A}$ receptor and brain-derived neurotrophic factor valine 66 to methionine genotypes impacts emotional brain and arousal correlates of risk for depression. Biol Psychiatry 2010; 68: 818-824.

34 Williams LM, Gatt JM, Schofield PR, Olivieri G, Peduto A, Gordon E. 'Negativity bias' in risk for depression and anxiety: brain-body fear circuitry correlates, 5-HTT-LPR and early life stress. Neuroimage 2009; 47: 804-814.

35 Heim C, Binder EB. Current research trends in early life stress and depression: review of human studies on sensitive periods, gene-environment interactions, and epigenetics. Exp Neurol 2012; 233: 102-111.

36 McGee RA, Wolfe DA, Yuen SA, Wilson SK, Carnochan J. The measurement of maltreatment: a comparison of approaches. Child Abuse Negl 1995; 19: 233-249.

37 Kaffman A, Meaney MJ. Neurodevelopmental sequelae of postnatal maternal care in rodents: clinical and research implications of molecular insights. J Child Psychol Psychiatry 2007; 48: 224-244.

38 Gatt JM, Nemeroff CB, Dobson-Stone C, Paul RH, Bryant RA, Schofield PR et al. Interactions between BDNF Val66Met polymorphisms and early life stress predict brain and arousal pathways to syndromal depression and anxiety. Mol Psychiatry 2009; 14: 681-695.

39 McEwen BS. Brain on stress: how the social environment gets under the skin. Proc Natl Acad Sci USA 2012; 109(Suppl 2): 17180-17185.

40 Camardese G, Di Giuda D, Di Nicola M, Cocciolillo F, Giordano A, Janiri L et al Imaging studies on dopamine transporter and depression: a review of literature and suggestions for future research. J Psychiatr Res 2014; 51: 7-18.

41 Oswald LM, Wand GS, Kuwabara H, Wong DF, Zhu S, Brasic JR. History of childhood adversity is positively associated with ventral striatal dopamine responses to amphetamine. Psychopharmacology 2014; 231: 2417-2433.

42 Baum N, Rahav G, Sharon M. Heightened susceptibility to secondary traumatization: a meta-analysis of gender differences. Am J Orthopsychiatry 2014; 84: 111-122.

43 Hardt J, Rutter M. Validity of adult retrospective reports of adverse childhood experiences: review of the evidence. J Child Psychol Psychiatry 2004; 45: 260-273. International License. The images or other third party material in this article are included in the article's Creative Commons license, unless indicated otherwise in the credit line; if the material is not included under the Creative Commons license, users will need to obtain permission from the license holder to reproduce the material. To view a copy of this license, visit http://creativecommons.org/licenses/ by/4.0/ 